\title{
Understanding Nickel Thin Film crystallization using X-Ray Diffractometry
}

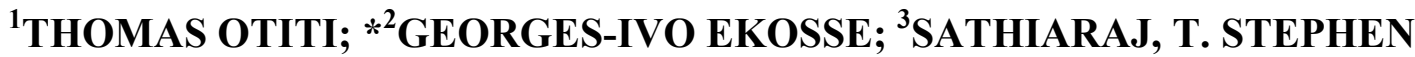

\author{
${ }^{* 1}$ Department of Physics, Makerere University, Box 7062, Kampala, Uganda. \\ ${ }^{2}$ Geology, Mining and Minerals programs, University of Limpopo, P/Bag X1106 Sovenga 0727, South Africa \\ ${ }^{3}$ Department of Physics, University of Botswana, 0022 Gaborone, Botswana
}

\begin{abstract}
Normal, helical and zigzag deposited Ni films were produced by letting a vapour stream of source material impinge on Corning 1737 glass substrates at oblique incidence while rotating the substrate during deposition. Films produced by glancing angle deposition (GLAD) technique while rotating the substrate. The microstructures of these Ni films were studied using X-ray diffractometry technique. The X-ray diffraction (XRD) patterns depicted $100 \%$ and $42 \%$ relative intensity (RI) peaks identified for normal and helical deposited $\mathrm{Ni}$ films but none for the zigzag deposited Ni film. Higher degree of crystallinity of Ni was demonstrated by the helical thin film sample having $200 \mathrm{~nm}$ thickness (sample Ni40) compared to the normal thin film which had only the $100 \%$ RI peak defined. Should an application therefore require $\mathrm{Ni}$ thin films of high crystallinity, it would be the film prepared with helical microstructure of $200 \mathrm{~nm}$ thickness that will be employed. @JASEM
\end{abstract}

Geologically, nickel occurs either as residual concentrations of nickeliferous laterites associated with basic/ultra basic igneous rocks, or nickel sulphide ores formed by replacement or magmatic injection (PIRSA Minerals, 2007). Sixty percent of $\mathrm{Ni}$ resources are of nickeliferous laterites and the rest of the sulphide. The nickel sulphide ores are associated with the platinum group metals (PGMs). There are approximately 30 minerals (Nickel Minerals, 2007) which contain Ni of which the most abundant is pentlandite. Its extraction and purification, and applications constitute a huge multi billion dollar industry. Its applications stretch into stainless steel, coinage, magnets, special alloys, painting and tinting, and laboratory uses (Nickel, 2007; Nickel Mineral Data, 2007).

An area in which $\mathrm{Ni}$ has found applications is the usage of thin films, which are essential in modern technologies and their optimization is the focus of continued growing research. Its application in thin films promotes the fabrication of nanostructured materials. By altering columnar inclination angle $(\beta)$, and vapour incidence angle $(\alpha)$, different and varied properties of the films emerge which are characteristic for special applications. Other factors, which affect the film structure, include material and deposition conditions such as substrate temperature, angular distribution of the deposition flux, background gas pressure and composition, and flux energies. Deposition patterns of films could be normal, helical or zigzag. These patterns coupled with the listed factors herein influence degree of crystallization; and hence determine application of product.

In varying these factors, and controlling the column angle $(\beta)$ and the flux angle $(\alpha)$ the density will vary in a predictable but not independently controllable or desirable manner. This direct dependence on flux angle $(\alpha)$ of both column angle $(\beta)$ and film density restricts the range of thin film microstructure attainable. For example, a porous film with vertical microstructure would be forbidden. The limitation could be overcome by producing $\mathrm{Ni}$ films using glancing angle deposition (GLAD) (Robbie et al., 1997). This technique combines oblique deposition onto a flat substrate with computer control of substrate rotation, enabling the column angle and film porosity to be chosen independently by rotating the substrate in a controlled manner. With control of microstructure, material properties for optical, chemical, biological, mechanical, and electrical applications can be tailored to a much higher degree of accuracy.

Being a novel technique of thin film deposition, demonstrated applications are increasingly developed. Some already feasible usages include magnetic media high density information storage (Hsieh et al., 1997), liquid crystal display technology (Robbie et al., 1999), photonic crystal (Kennedy et al., 2003), optical rotators, polarization beam splitters (Azzam, 1992), optical filters (Hodgkinson et al., 2000; Hodgkinson et al., 2000a; Kaminska et al., 2003). The technique is being explored for applications in the bioluminescence sensors, electroluminescent devices, optical transparent conducting films from pure metals, multistate electronic switches based on filamentary conduction, optical sensors that can detect and quantify various chemical and biological fluids, microsieves for entrapment of viruses, porous materials for growing biological tissues, chemical sensors, catalytic reaction surfaces, optical coatings, thermoelectric materials, quantum effect devices, field emitters, and solar cells.

Different analytical tools have been used in characterizing thin films (Murakami et al., 1996; Bueno-Core et al., 2004; Noh et al., 2000). One of such tool is the X-ray diffractometry. The XRD 
technique aids in finger printing phases present in an analyte by identifying and characterizing it. There is increasingly wide significance and promising applications of $\mathrm{Ni}$ thin films and the potential for those particularly produced from GLAD technique to gain strong industrial applications. This study thus employed X-ray diffractometry in understanding Ni thin films crystallization. It is anticipated that the generated knowledge will form basis for further applications of $\mathrm{Ni}$ thin films.

\section{MATERIALS AND METHODS}

Film preparation: Four different samples of Ni films (samples Ni0, Ni40, Ni80 and Ni70) were prepared by GLAD technique by evaporating $\mathrm{Ni}$ wire onto glass substrates in a vacuum chamber at pressures not greater than $10^{-7} \mathrm{mb}$ at room temperature. Corning 1737 glass substrates were cleaned and attached to substrate holder in the chamber. The substrate was tilted and rotated as desired. The tilt gave the vapour incidence angle to the substrate normal. The rotation of the substrate was computer controlled to give the desired shape of the microstructure. Stepper motors controlled the deposition angle, $(\alpha$ tilt $)$ and rotation of the substrate about an axis normal to its surface ( $\phi$ rotation). A computer based feedback control system actuates the motors in accordance with parameterized programs of $\alpha$ and $\phi$ as functions of accumulated film thickness. By moving the substrate in a controlled way during deposition, the shape of the columns was accurately tailored on a nanometer scale. The columnar morphology was therefore directly related to the dynamically controlled angular coordinates $(\alpha, \phi)$ of the vapour deposition source

Sample Ni0 was prepared with the glass substrate horizontal; with the vapour incident angle to the substrate normal being $0^{\circ}$ and the film thickness 300 nm. Sample Ni40 was prepared with the glass substrate tilted; with the vapour incident angle to the substrate normal being $40^{\circ}$ and the film thickness 200 $\mathrm{nm}$ from two turns of a $100 \mathrm{~nm}$ each. It was helical in microstructure with two rotations. Sample Ni80 was prepared with the glass substrate tilted; with the vapour incident angle to the substrate normal being $80^{\circ}$ and the film thickness $200 \mathrm{~nm}$ from two turns of a $100 \mathrm{~nm}$ each. It was also helical in microstructure with two rotations. Sample Ni70 was prepared with the glass substrate tilted; with the vapour incident angle to the substrate normal being $70^{\circ}$ and the film thickness $1000 \mathrm{~nm}$ from 10 zigzags. Its microstructure was zigzag.

X-Ray Diffraction Analysis: The X-ray diffractometer (Philips PW 3710 XRPD system) used for the analyses of the samples operated at $40 \mathrm{kV}$ and 45
$\mathrm{mA}$, with a $\mathrm{Cu}-\mathrm{Ka}$ radiation and a graphite monochromator with PW 1877 Automated Powder Diffraction. A 2001 X'PERT Data Collector software package was used for capturing of phases present in the different samples. The X-rays were produced at the wavelengths $\lambda_{1}=1.54056 \AA$ and $\lambda_{2}=1.54439 \AA$ with $\lambda_{2}$ stripped off. Samples were scanned from $2^{\circ}$ $2 \theta$ to $80^{\circ} 2 \theta$ using a step size of $2 \theta=0.02$ and a time per step of 0.30 seconds and their diffractograms were recorded. A 2001 version of the Philips X'PERT graphics \& Identify software package was used for qualitative identification of the minerals from both the data and patterns obtained by scanning. The interpreted results were compared with data and patterns available in the mineral Powder Diffraction File data book and search manual issued by the International Centre for Diffraction Data (2001) for confirmation.

\section{RESULTS AND DISCUSSION}

Figures 1 to 4 are the X-ray diffractograms of the samples, and Table 1 gives their peak properties according to peak angle, peak height and peak tip width. The ideal $100 \% \mathrm{RI}$ peak for $\mathrm{Ni}$ is at $44.52 \theta$, and other significant peaks for $\mathrm{Ni}$ identification are the $42 \% \mathrm{RI}$ at 51.820 , and $21 \% \mathrm{RI}$ at 76.320 . Sample Ni0 was crystallined with only the $100 \%$ RI peak very well defined. Sample Ni40 was crystallined with both the $100 \%$ and $42 \%$ RI peaks very well defined. Whereas sample Ni70 was amorphous with not a single defined peak, sample Ni80 was poorly crystallined with its peaks not clearly defined.

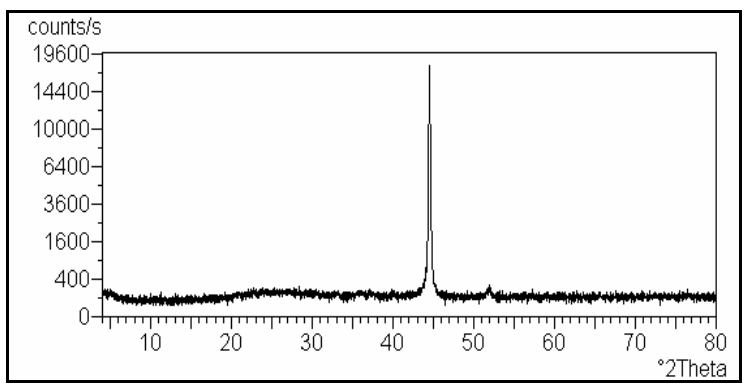

Fig 1: X-ray diffractogram of normal deposited $\mathrm{Ni}$ thin film (sample $\mathrm{NiO})$

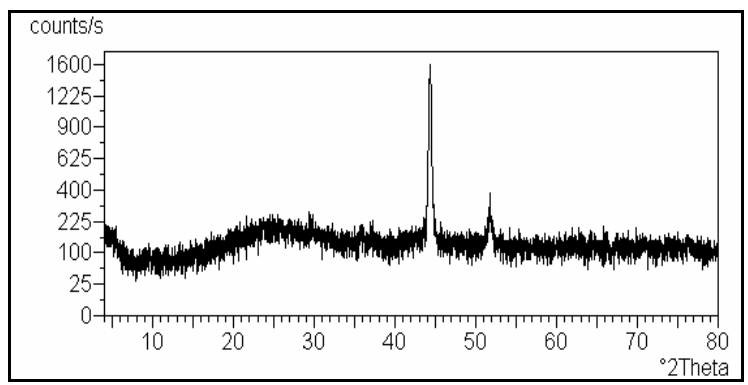


Fig 2: X-ray diffractogram of helical deposited Ni thin film (sample Ni40)

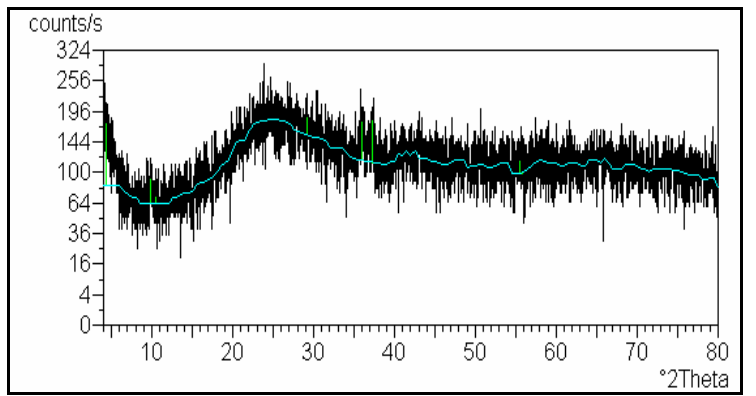

Fig 3: X-ray diffractogram of zigzag deposited $\mathrm{Ni}$ thin film (sample Ni70)

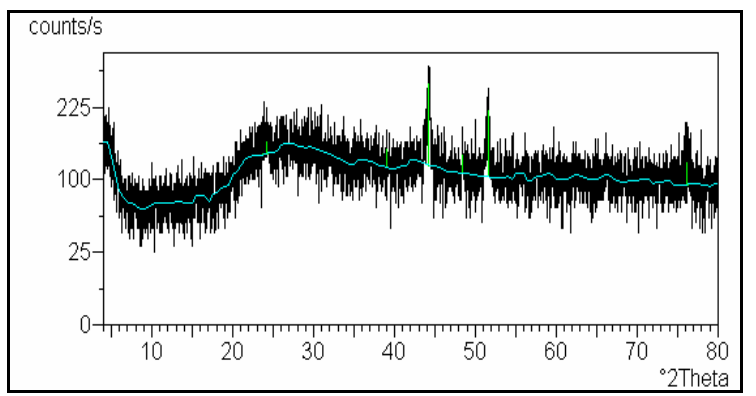

Fig 4: X-ray diffractogram of helical deposited Ni thin film (sample Ni80)

Table 1: Peak characteristics obtained from X-ray diffractometry of samples of Ni thin films

\begin{tabular}{cccccc}
\hline S/ & $\begin{array}{c}\text { Peak } \\
\text { angle } \\
(2 \theta\end{array}$ & $\begin{array}{c}\text { Relative } \\
\text { intensity } \\
(\%)\end{array}$ & $\begin{array}{c}\text { Miller } \\
\text { indices } \\
(\mathrm{hk} l)\end{array}$ & $\begin{array}{c}\text { Peak } \\
\text { height } \\
\text { (count } \\
\text { angle) }\end{array}$ & $\begin{array}{c}\text { Peak } \\
\text { tip } \\
\text { width } \\
(2 \theta)\end{array}$ \\
Ni0 & 44.5 & 100 & 111 & 1798.4 & 0.10 \\
& 51.9 & 42 & 200 & 161.9 & 0.16 \\
Ni40 & 44.4 & 100 & 111 & 1440.9 & 0.18 \\
& 51.7 & 42 & 200 & 158.4 & 0.40 \\
Ni70 & - & & & - & - \\
Ni80 & 44.3 & 100 & 111 & 156.3 & 0.24 \\
& 51.8 & 42 & 200 & 113.9 & 0.20 \\
& 76.2 & 21 & 220 & 31.3 & 0.64 \\
\hline
\end{tabular}

Using glancing angle deposition, porous chiral and zigzag Ni films with tailourable microstructure were fabricated. With this technique of growth during spinning of the substrate and growth while the substrate is stationary, column angle and azimuthal orientation can be controlled independent of film density. Film density is then specified by choosing the oblique angle of vapour flux incidence at the substrate. Helical and zigzag structures with modified geometry have been demonstrated.

In understanding the crystallinity of the Ni thin films, $\mathrm{X}$-ray diffrctometry was employed. The X-ray diffractometry thus demonstrated that $\mathrm{Ni}$ thin film prepared by GLAD in which the glass substrate is tilted, and having a vapour incident angle to the substrate normal being $40^{\circ}$ and the film thickness 200 coupled with a helical microstructure; had higher crystallinity compared to that prepared normally. This GLAD technique therefore is suitable for the production of $\mathrm{Ni}$ thin films, and the characterization. It defines with the aid of X-ray diffractometry how thin films of high crystallinity could be produced with significant accuracy.

Acknowledgement: The authors thank Prof. K. Robbie of Queen's University, Canada for preparation of the samples used in this study. One of us (T.O) would like to thank University of Botswana, Botswana for offering him a position of visiting scholar, and Makerere University for granting him leave to take the offer. The financial support to visit University of Botswana has been provided by the International Science Programme (ISP), Uppsala University, Sweden and African Network of Scientific and Technological Institutions (ANSTI), UNESCO Nairobi Office.

\section{REFERENCES}

Azzam R. M. A. (1992) Chiral thin solid films: Method of deposition and applications. Applied Physics Letters 61 (26), 3118-3120

Bueno-Core G. E., Tejos M., Schrebler R., Klahn A. H. and Hill R. H. (2004) Nickel tropolonate complexes as precursors for the direct photodeposition of $\mathrm{NiO}$ thin films. Journal of Chilean Chemical Society 49 (3), 223226.

Hsieh Y. C., Gadetsky S., Suzuki T., and Mansuripur M. (1997) Oblique sputtering of amorphous $\mathrm{TbFeCo}$ thin films on glass substrates and the effect of deposition angle on perpendicular magnetic anisotropy. Journal of Applied Physics 81 (8,) 3555-3560

Hodgkinson I. J., Lakhtakia A. and Wu Q. H (2000) Experimental realization of sculptured-thinfilm polarization-discriminatory lighthandedness inverters. Optical Engineering 39, 2831-2834.

Hodgkinson I. J., Wu Q. H., Thorn K. E., Lakhtakia A. and McCall M. W. (2000) Spacerless circular-polarization spectral-hole filters using chiral sculptured thin films: theory and experiment, Optics Communications, 184 (14), 57-66.

International Centre for Diffraction Data (2001) International Centre for Diffraction Data. Mineral Powder Diffraction File Databook. Pp 942. 
Kaminska K., Brown T., Beydagghyan G. and Robbie K. (2003) Vacuum Evaporated Porous Silicon Photonic Interference Filters. Applied Optics 42, 4212-4219.

Kennedy S. R., Brett M. J., Miguez H., Toader O. and John S. (2003) Optical properties of a three-dimensional silicon square spiral photonic crystal. Photonics and Nanostructures 1, 37-42

Murakami Y., Kuratani N., Imai O. and Ogata K. (1996) Study on the crystallization of nickel films prepared by the ion beam and vapour deposited method. European Materials Research Society 1995 Spring Conference, Symposium E: Structures and properties of metalic thin films and multilayers, 275 (1-2) 61-63.

Nickel (2007) Nickel - Wikipedia, the free encyclopedia. http://en.wikipedia.org/wiki/Nickel. Accessed 27 April 2007.

Nickel Minerals (2007) Category: Nickel minerals. Wikipedia, the free encyclopedia. http:/en.wikipedia.org/wiki/Category:Nickel_mi nerals. Accessed 28 April 2007.
Nickel Mineral Data (2007) Nickel mineral data. http://webmineral.com/data/Nickel.shtml.

Accessed 28 April 2007.

Noh H. Y., Lee K. H., Cul X. X. and Choi C-S. (2000) The composition and structure of TiNi thin film formed by electron beam evaporation. Scripta Materialia 43(9) 847-852.

PIRSA Minerals (2007) Nickel. Mineral Resource Potential $\quad-\quad$ Nickel. http://www.pir.sa.gov.au/pages/minerals/commo dity/nickel.htm. Accessed 27 April 2007.

Robbie K., Hnatiw A. J. P., Brett M. J., MacDonald R. I. and McMullin N. J. (1997) Inhomogeneous Thin Film Optical Filters Fabricated Using Glancing Angle Deposition. Electronics Letters 33(14) 1213-1214.

Robbie K. Broer D.J. Brett M.J (1999) Chiral nematic order in liquid crystals imposed by an engineered inorganic nanostructure. Nature 399, 764-766. 